\title{
Lidil
}

Revue de linguistique et de didactique des langues

Multimodalité de la communication chez l'enfant

\section{Le développement des gestes pragmatiques et leur relation avec le développement de la compétence textuelle chez l'enfant âgé de 4 à 10 ans}

Maria Graziano

\section{OpenEdition}

Journals

Édition électronique

URL : http://journals.openedition.org/lidil/3076

DOI : 10.4000/lidil.3076

ISSN : 1960-6052

Éditeur

UGA Éditions/Université Grenoble Alpes

Édition imprimée

Date de publication : 30 novembre 2010

Pagination : 113-138

ISBN : 978-2-84310-184-7

ISSN : 1146-6480

Référence électronique

Maria Graziano, «Le développement des gestes pragmatiques et leur relation avec le développement de la compétence textuelle chez l'enfant âgé de 4 à 10 ans », Lidil [En ligne], 42 | 2010, mis en ligne le 31 mai 2012, consulté le 19 avril 2019. URL : http://journals.openedition.org/lidil/3076 ; DOI :

10.4000/lidil.3076 


\title{
Le développement des gestes pragmatiques et leur relation avec le développement de la compétence textuelle chez l'enfant âgé de 4 à 10 ans
}

\author{
Maria Graziano*
}

RÉSUMÉ

Le geste est une composante importante de la communication en coprésence qui se développe de manière analogue aux habiletés linguistiques. Cette étude est centrée sur la relation entre l'évolution de la compétence textuelle et le développement de la capacité à utiliser les gestes pragmatiques avec les trois fonctions (performative, modale, de parsing) distinguées par Kendon (2004). Trente-trois enfants italiens âgés de 4 à 10 ans ont été enregistrés tandis qu'ils produisaient le récit d'un dessin animé. La composante linguistique a été examinée du point de vue du développement des compétences syntaxiques (nombre et types de propositions) et textuelles (usage de différents types de connecteurs). Les gestes ont été classés tout d'abord entre référentiels et pragmatiques, puis à l'intérieur de cette dernière catégorie entre gestes «performatives», «modals» et de «parsing». Les résultats, obtenus aux plans qualitatif et quantitatif, montrent l'existence d'une étroite corrélation entre le développement de ces gestes, l'évolution de la complexité syntaxique et l'usage de différents types de connecteurs liée à l'accroissement des habiletés textuelles et pragmatico-communicatives de l'enfant.

\section{ABSTRACT}

Gesture is an important component of communication in social interactions, that develops in parallel to the linguistic abilities. This study is focused on the relationship between the development of textual competence and the capacity to use the three functions of pragmatic gestures (performative, modal and parsing) identified by Kendon (2004). A group of 33 Italian children were videotaped while producing the

* University of Alberta, Edmonton (Canada). 
narration of a cartoon. The verbal component was examined in terms of syntactical (number and types of clauses) and textual (usage of different types of connectives) competences. Pragmatic gestures were first distinguished from representatives and then, were further divided into performatives, modals and parsing. The results, obtained both from quantitative and qualitative analyses, showed a tight correlation between the development of these gestures and textual competence, particularly syntactical complexity and the usage of different types of connectives. These results suggest that pragmatic-communicative abilities develop simultaneously in gestures and speech.

Depuis la seconde moitié du $\mathrm{xx}^{\mathrm{e}}$ siècle, on a commencé à s'intéresser aux composantes kinésiques qui, outre la langue, participent au processus de la communication pendant l'interaction en face-à-face : les gestes, les expressions faciales, les conduites proxémiques, les regards.

Ce sont surtout les gestes qui ont fait l'objet de nombreuses investigations, grâce aux recherches de Kendon $(1972,1980,2004)$ et de McNeill $(1992,2000)$ qui considèrent gestes et paroles comme deux aspects d'un seul processus d'élaboration et de communication du message. Cette thèse s'appuie sur l'observation de l'étroite intégration rythmique et communicative entre gestes et paroles, qui se manifeste tant dans la fonction référentielle que dans la fonction pragmatique du discours.

Sur ce postulat de la multimodalité du message se fonde l'hypothèse selon laquelle l'acquisition du code verbal et l'acquisition du code gestuel procèderaient d'un même processus d'apprentissage. Une série de travaux ont permis de comprendre comment la relation entre gestes et paroles se constitue et évolue, mettant en lumière d'une part, le rôle considérable que les gestes revêtent dans ce processus, et de l'autre, d'importants phénomènes parallèles dans le développement de ces deux modalités de l'expression.

On a montré que le lien entre parole et geste émerge dans les premiers stades de l'évolution de l'enfant (Iverson et Thelen, 1999; Iverson et Fagan, 2004) et que le geste, en particulier celui de pointage, constitue la première forme de communication intentionnelle de l'enfant (Bates et al., 1975, 1979; Liszkowski, 2005). Il reste, en outre, la forme d'expression dominante jusqu'à 20 mois, âge où l'on assiste à un accroissement de l'usage des unités linguistiques (Iverson et al., 1994). De plus, on a observé que les premiers énoncés composés de deux unités sont constitués par des énoncés crossmodals qui combi- 
nent un mot et un geste, qui se différencient dans la forme et qui sont prédictifs du développement verbal ultérieur de l'enfant (Iverson et al., 1994 ; Capirci et al., 1996, 2005 ; Butcher et Goldin-Meadow, 2000 ; Özçalişkan et Goldin-Meadow, 2005 ; Pizzuto et al., 2005).

Bien que moins riches, les recherches menées sur les enfants âgés de plus de trois ans ont prouvé que la relation entre geste et parole continue à évoluer. On a démontré qu'à l'évolution des capacités cognitives, discursives et communicatives correspond un développement du système gestuel en termes de variété de types de gestes produits, de degré d'abstraction et de contenus communicatifs exprimés par ceux-ci (McNeill, 1992; Colletta, 2004, 2009; Cristilli et al., à paraitre).

S'agissant des gestes représentatifs, par exemple, les premiers à apparaitre après le geste de pointage, McNeill (1992) a illustré que de manière analogue à ce qui se passe sur le plan linguistique, sur le plan gestuel aussi on assiste à un processus qui va de formes très concrètes, iconiques et idiosyncrasiques de représentation, à des formes de plus en plus symboliques et standardisées. Plus récemment, Capirci et al. (à paraitre) ont montré que ce type de gestes évolue également en termes de précision formelle et de clarté représentative.

On a montré, en outre, qu'en fonction de l'âge non seulement les gestes accompagnent de plus en plus la production verbale, mais que parallèlement à l'évolution des habiletés discursives et textuelles le répertoire des gestes s'enrichit. On observe ainsi l'apparition de gestes qui expriment des contenus plus abstraits (les métaphoriques de McNeill), et l'usage de gestes à fonction pragmatique et textuelle que les enfants les plus âgés utilisent pour signaler la structuration du texte et des commentaires (Colletta, 2004, 2009).

L'étude qu'on présente ici vise à approfondir la relation entre l'évolution de la compétence textuelle et le développement des gestes pragmatiques produits dans l'élaboration d'un discours narratif. Plus précisément, notre étude est centrée sur le développement de la capacité à utiliser les gestes pragmatiques avec les trois fonctions (performative, modale, de parsing) distinguées par Kendon (2004), dans le but de montrer l'existence d'une corrélation entre le développement de ces gestes et l'évolution de certaines habiletés linguistiques qui manifestent la compétence textuelle : la complexité syntaxique et l'usage de différents types de connecteurs. 


\section{Méthode}

\section{Corpus et procédure}

Les narrations d'un bref dessin animé, préalablement montré deux fois, constituent le corpus de cette recherche dont les participants sont trentetrois enfants italiens âgés de 4 à 10 ans et répartis en trois groupes d'âge (tableau 1).

\begin{tabular}{|c|c|c|c|}
\hline Groupe & N.participants & Range & Âge moyen \\
\hline I & 11 & $4,1-5,4$ & 4,7 \\
\hline II & 11 & $6-7,8$ & 6,8 \\
\hline III & 11 & $8-10,9$ & 9,2 \\
\hline
\end{tabular}

Tableau 1. - Participants répartis par tranches d'âge.

Le dessin animé appartient à la série télévisée Pingu, ayant comme protagonistes une famille de pingouins qui ne parlent pas mais produisent des sons indistincts. Dans l'épisode choisi, les personnages se consacrent aux préparatifs pour Noël : préparation des biscuits, décoration de l'arbre de Noël et empaquetage des cadeaux. Tous les enfants ont accompli la tâche narrative avec un adulte qu'ils connaissaient déjà, et dans un contexte familial. Pendant la narration, l'adulte, qui connaissait l'histoire, n'interrompait pas l'enfant mais l'aidait en cas d'évidente difficulté, en posant des questions neutres, comme «qu'est-il arrivé après?». Chaque enfant a été enregistré seul et en une seule fois.

\section{Codage des phénomènes linguistiques}

La production verbale des enfants a été segmentée en propositions. Pour l'évaluation de la complexité syntaxique, on a considéré les relations de subordination et de coordination. Les propositions ont été codifiées en trois catégories : indépendantes, principales et subordonnées. Ensuite, pour chaque catégorie, on a distingué entre simple, si la proposition ne présentait aucun rapport de coordination avec d'autres propositions, et coordonnée, si la proposition était introduite par une conjonction de coordination qui la reliait à d'autres propositions.

Les connecteurs ont été classés selon une taxinomie de type fonctionnelle (tableau 2), élaborée sur la base de plusieurs lectures (Halliday et Hasan, 1976; Lundquist, 1994; Bazzanella, 1991, 1994; Berretta, 
1984 ; Roulet et al., 1985 ; Simone, 1990; Chini et al., 2003) et dans laquelle on a inclus plusieurs éléments linguistiques (conjonctions de coordination et de subordination, prépositions, adverbes, signaux discursifs, interjections).

\begin{tabular}{|c|c|}
\hline $\begin{array}{l}\text { Catégories des connecteurs identifiées sur la } \\
\text { base de la relation cohésive qu'ils expriment }\end{array}$ & Formes \\
\hline Temporels & puis, après, tandis que, etc. \\
\hline $\begin{array}{c}\text { Causatifs } \\
\text { (Causatifs / Consécutifs / Finaux) }\end{array}$ & parce que, donc, pour, etc. \\
\hline Adversatifs & mais, par contre, etc. \\
\hline Additifs & et, aussi, etc. \\
\hline Explicatifs & en effet, c'est-à-dire, etc. \\
\hline $\begin{array}{c}\text { Métatextuels } \\
\text { (Démarcatifs / Reformulatifs) }\end{array}$ & $\begin{array}{l}\text { alors, ça suffit/ je veux dire, } \\
\text { disons, etc. }\end{array}$ \\
\hline $\begin{array}{c}\text { Interactifs } \\
\text { (Éléments de remplissage/Éléments aptes } \\
\text { à contrôler la réception/Mécanismes de } \\
\text { modulation) }\end{array}$ & $\begin{array}{l}\text { disons/n'est-ce pas? / il me } \\
\text { semble, etc. }\end{array}$ \\
\hline
\end{tabular}

Tableau 2. - Typologie des connecteurs.

\section{Codage des gestes}

La production gestuelle des enfants a été analysée en termes fonctionnels. Les gestes identifiés ont été distingués entre référentiels et pragmatiques. Kendon (2004, p. 159-160) définit comme référentiels les gestes qui contribuent à exprimer un aspect du contenu propositionnel de l'énoncé, c'est-à-dire les gestes qui fournissent une représentation du référent, en traçant la forme ou la dimension d'un objet ou d'une personne, ou en mimant un comportement ou une action (gestes représentationnels), aussi bien que les gestes qui désignent le référent au moyen d'une indication de la main, de l'index ou d'autres parties du corps (gestes déictiques). Par contre, les gestes pragmatiques sont définis comme des gestes qui expriment des aspects du contenu de l'énoncé qui ne sont pas liés à son signifié référentiel (Kendon, 2004, p. 158). En suivant l'auteur, dans cette catégorie, on a distingué entre gestes «performatives», «modals» et «parsing». Les premiers sont des gestes qui réalisent un acte communicatif, comme acquiescer ou nier avec la tête ou présenter le discours à l'interlocuteur à l'aide d'un geste descendant 
réalisé la main ouverte, paume vers le haut. Les deuxièmes sont des gestes qui fournissent la clé d'interprétation de l'énoncé, comme ajouter l'idée de négation, non exprimée dans l'énoncé, par un geste latéral réalisé les mains ouvertes, paumes vers le bas. Les troisièmes, enfin, sont des gestes qui contribuent au processus de structuration du discours en marquant les parties dont il est composé, comme le geste qui est réalisé avec le pouce et l'index qui se touchent en formant un cercle et qui sert à préciser ou accentuer certaines unités du discours (voir Kendon, 2004, p. 238-247) ${ }^{1}$.

L'analyse des phénomènes apparus dans les deux modalités a été menée du point de vue qualitatif et quantitatif.

\section{Résultats}

\section{Analyse quantitative et qualitative de la complexité syntaxique}

L'analyse de la composante verbale a montré, en premier lieu, qu'en fonction de l'âge, la longueur moyenne des narrations augmente progressivement, en passant presque du simple au double (tableau 3). Ce premier résultat apparait cohérent avec le développement de la capacité à assumer seul l'activité langagière et à produire un discours monologué (en l'espèce narratif) [Berman et Slobin, 1994].

\begin{tabular}{|c|c|}
\hline Groupe & $\begin{array}{c}\text { Nombre moyen des } \\
\text { propositions }\end{array}$ \\
\hline I & 20,7 \\
\hline II & 34,9 \\
\hline III & 38,7 \\
\hline
\end{tabular}

Tableau 3. - Nombre moyen de propositions produites dans chaque groupe.

1. Il convient de préciser qu'il s'agit d'une typologie des fonctions et non pas des gestes (comme Kendon (2004) le met bien en évidence), car différentes expressions gestuelles peuvent avoir une des fonctions susmentionnées. De plus, un même geste peut avoir à la fois une fonction performative, modale ou de parsing, selon les contextes discursifs dans lesquels il est utilisé. En outre, dans plusieurs cas, les gestes peuvent avoir plusieurs fonctions en même temps (Kendon, 2004; McNeill, 1992). La classification de chaque geste a donc été établie sur la base de la fonction qui semblait dominante. 
L'examen des types de propositions produites par les trois groupes d'enfants a permis de mettre en évidence une complexification graduelle de la structure syntaxique (tableau 4). De fait, on assiste à la diminution progressive, en pourcentage, de l'emploi des indépendantes au profit des principales et, plus encore, des subordonnées.

\begin{tabular}{|c|c|c|c|}
\hline Groupe & Indépendantes & Principales & Subordonnées \\
\hline I & $79,2 \%$ & $10 \%$ & $10,9 \%$ \\
\hline II & $73,5 \%$ & $12,1 \%$ & $14,4 \%$ \\
\hline III & $67,8 \%$ & $14,7 \%$ & $17,5 \%$ \\
\hline
\end{tabular}

Tableau 4. - Pourcentage des trois types de propositions par rapport au total de celles produites dans chaque groupe.

Ces données quantitatives sont étayées par l'analyse qualitative des types de structures complexes identifiées dans les textes examinés. On a constaté, par exemple, que les propositions subordonnées du second degré ${ }^{2}$ étaient présentes exclusivement dans les récits des groupes II et III. Un autre phénomène concerne l'usage des propositions enchâssées qui, bien que relevées dans tous les groupes, montrent des différences qui reposent sur la nature des propositions qui sont insérées dans la principale. De fait, les enfants entre 4 et 5 ans se sont limités à la production des relatives, tandis que les enfants les plus âgés ont produit des subordonnées conjonctives de but ainsi que des temporelles. Cette plus grande diversité s'explique par l'évolution de la capacité à comprendre et à expliciter les différents types de relations existant parmi les évènements narrés, comme on le verra dans la prochaine section.

Bien que les différences dans le degré de complexité syntaxique des récits examinés aient été observées principalement sur la base de la présence des structures subordonnées, l'occurrence des liens de coordination a paru être un autre aspect à ne pas négliger, puisque la coordination aussi est une manifestation de ce qu'on appelle la «syntaxe complexe» (Tomasello, 2003). Afin d'évaluer, donc, l'incidence des structures coordonnées au sein de chaque catégorie de propositions (indépendantes, principales et subordonnées) on a distingué les propositions simples et les propositions coordonnées.

2. Propositions subordonnées qui dépendent d'une autre subordonnée. 
La comparaison de l'usage des structures simples et coordonnées dans les trois groupes est présentée dans la figure 1, tandis que le tableau 5 montre les pourcentages des trois types de propositions distinguées entre simples et coordonnées par rapport au total de chaque catégorie.

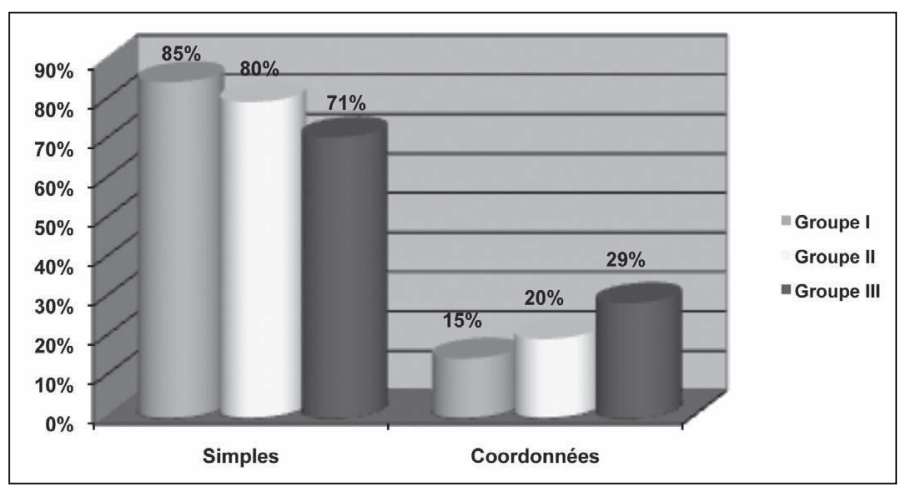

Figure 1. - Pourcentage des propositions simples et coordonnées par rapport au total des propositions produites dans chaque groupe.

\begin{tabular}{|c|c|c|c|c|c|c|}
\hline Groupe & $\begin{array}{c}\text { Ind } \\
\text { Simp / } \\
\text { Ind }\end{array}$ & $\begin{array}{c}\text { Ind } \\
\text { Coor / } \\
\text { Ind }\end{array}$ & $\begin{array}{c}\text { Princ } \\
\text { Simp / } \\
\text { Princ }\end{array}$ & $\begin{array}{c}\text { Princ } \\
\text { Coor / } \\
\text { Princ }\end{array}$ & $\begin{array}{c}\text { Sub } \\
\text { Simp / } \\
\text { Sub }\end{array}$ & $\begin{array}{c}\text { Sub } \\
\text { Coor / } \\
\text { Sub }\end{array}$ \\
\hline I & $82 \%$ & $18 \%$ & $91 \%$ & $9 \%$ & $100 \%$ & $0 \%$ \\
\hline II & $77 \%$ & $23 \%$ & $83 \%$ & $17 \%$ & $93 \%$ & $7 \%$ \\
\hline III & $65 \%$ & $35 \%$ & $71 \%$ & $29 \%$ & $93 \%$ & $7 \%$ \\
\hline
\end{tabular}

Tableau 5. - Pourcentage des trois catégories de proposition distinguées entre simples (simp) et coordonnées (coor) par rapport au total de chaque catégorie.

Comme la figure 1 le met clairement en évidence, et de manière analogue à ce qu'on a observé concernant la production des propositions subordonnées, la proportion des coordonnées augmente elle aussi progressivement en fonction de l'âge, atteignant dans le groupe III un pourcentage pratiquement double par rapport à celle du groupe I (29\% vs $15 \%$ ); cela au détriment des propositions simples qui diminuent en pourcentage. De plus, comme le montrent les résultats reproduits dans le tableau 5, il est aussi intéressant de souligner que, au fil de l'âge, on observe une habileté croissante à coordonner entre elles non seulement 
les propositions indépendantes, mais aussi les principales (exemple 1 ci-après) ainsi que, à partir du groupe intermédiaire, les subordonnées (exemple 2 ci-après) ${ }^{3}$.

(1)

\begin{tabular}{|ll|}
\hline Viola (Groupe II) \\
$15 \quad \begin{array}{l}\text { elle les ferme avec la clé les fils } \\
\text { li chiude a chiave ai figli (princ simple) } \\
\text { et met une boule }\end{array}$ \\
$16 \quad \begin{array}{l}\text { e li mette la palla (princ coor) } \\
\text { pour qu'ils ne regardent pas } \\
\text { per non farli sbirciare }\end{array}$ \\
\hline
\end{tabular}

(2)

\begin{tabular}{|ll|}
\hline Sonia (Groupe II) \\
5 & $\begin{array}{l}\text { la maman avait enfermé à l'intérieur les pingouins } \\
\text { la mamma aveva chiuso dentro i pinguini (princ) }\end{array}$ \\
6 & $\begin{array}{l}\text { parce qu'ils devaient faire l'arbre } \\
\text { perché dovevano fa' l'albero (sub simp) } \\
\text { et mettre les cadeaux dessous } \\
7\end{array}$ \\
\hline
\end{tabular}

Ces données fournissent donc une preuve supplémentaire de l'évolution de la capacité à construire des structures complexes.

\section{Analyse quantitative des connecteurs}

L'analyse des connecteurs a permis de mettre en évidence, tout d'abord, un accroissement de leur emploi (tableau 6), un résultat qui apparait cohérent avec l'augmentation des structures subordonnées et coordonnées que nous venons de décrire et qui sont justement réalisées au moyen de certains des outils cohésifs examinés dans cette section.

\begin{tabular}{|c|c|}
\hline Groupe & Taux des connecteurs \\
\hline I & $55,3 \%$ \\
\hline II & $74,9 \%$ \\
\hline III & $71,9 \%$ \\
\hline
\end{tabular}

Tableau 6. - Taux des connecteurs par rapport au total des propositions produites dans chaque groupe.

3. Les conventions de transcription verbale, tout comme celles de la transcription des gestes, sont décrites dans l'appendice. 
En ce qui concerne la distribution des connecteurs dans les différentes catégories fonctionnelles, l'ensemble des données indique que l'usage de chaque type de connecteurs tend à s'intensifier, à l'exception des temporels qui, au contraire, montrent une faible diminution du premier au troisième groupe (tableau 7).

\begin{tabular}{|c|c|c|c|c|c|c|c|}
\hline 芯 & 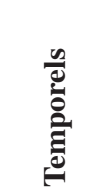 & 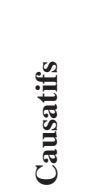 & 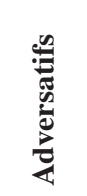 & 窇 & : & 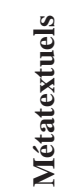 & 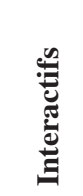 \\
\hline I & $27 \%$ & $3,7 \%$ & $3 \%$ & $17 \%$ & $0 \%$ & $3,3 \%$ & $1,4 \%$ \\
\hline II & $36,9 \%$ & $7 \%$ & $2,2 \%$ & $18,9 \%$ & $0,3 \%$ & $5,7 \%$ & $4,3 \%$ \\
\hline III & $25,4 \%$ & $6,2 \%$ & $4,7 \%$ & $24,9 \%$ & $1 \%$ & $5,2 \%$ & $5,2 \%$ \\
\hline
\end{tabular}

Tableau 7. - Taux des différents types de connecteurs par rapport au total des propositions produites dans chaque groupe.

Pour commenter ces résultats il faut rappeler que le type de texte que ces enfants ont produit (la narration) est un type de discours comprenant deux fonctions : la fonction référentielle et la fonction évaluative (Labov et Waletzky, 1967; Labov, 1972). La narration, en fait, est une activité sociale, caractérisée par une dimension interactive (Losh et al., 2000; Reilly et al., 2004) et produire un récit signifie à la fois ré-évoquer une série de faits et les interpréter et les évaluer. Il faut donc organiser le récit dans une structure précise, normalement constituée par une séquence d'épisodes et d'évènements qui se déroulent hiérarchiquement dans le temps et qui sont connectés entre eux par des relations logico-causales qui motivent le comportement des personnages (Mandler et Johnson, 1977; Stein et Glenn, 1979). En même temps, il faut fournir des évaluations sur les évènements, tout comme sur les comportements des personnages, en leur attribuant des buts et des intentions. De cette façon, le narrateur manifeste son point de vue sur ce qu'il est en train de dire, en maintenant l'attention de l'interlocuteur et en orientant son flux d'attention (Labov, 1972; Peterson et McCabe, 1983 ; Berman et Slobin, 1994).

Sur la base de ces caractéristiques du discours narratif, on peut dire que les résultats de l'analyse sont cohérents avec l'évolution des habiletés textuelles et pragmatico-communicatives qui président à la production d'une narration comprenant les deux fonctions susmen- 
tionnées. La diminution de l'incidence des connecteurs temporels peut être interprétée comme un indice du fait que la tendance à organiser le discours narratif en privilégiant la trame évènementielle décroit avec l'âge, pour laisser la place à l'expression des relations logico-causales, qui sont exprimées de plus en plus fréquemment dans les tranches d'âges supérieures (Peterson et McCabe, 1983; Berman et Slobin, 1994). L'accroissement des explicatifs, des métatextuels et des interactifs est à expliquer, par contre, par l'augmentation de la compétence pragmatique et communicative qui se développe elle aussi progressivement (Peterson et McCabe, 1983; Bamberg et Damrad-Frye, 1991; Reilly, 1992; Berman et Slobin, 1994 ; Bamberg et Reilly, 1996 ; Baumgartner et al., 2000 ; Losh et al., 2000 ; Reilly et al., 2004; Reig et Colletta, soumis). La forte incidence des connecteurs additifs dans tous les groupes s'explique enfin par le fait que cette fonction est exprimée à travers la conjonction «et», un connecteur très fréquent dans le langage enfantin et dans ce type de texte (Berman et Slobin, 1994; Peterson et McCabe, 1987).

\section{Analyse qualitative des connecteurs}

Cette interprétation des données quantitatives est confirmée par leur examen qualitatif qui montre une différentiation graduelle dans l'usage de tous ces types de connecteurs. Nous nous concentrons ici sur les constats issus de l'analyse des temporels, des métatextuels et des interactifs; les catégories qui, comme on le verra dans la section suivante, ont montré une plus stricte corrélation avec le développement des gestes pragmatiques.

Concernant les connecteurs temporels, on a constaté un enrichissement graduel du répertoire des formes utilisées dans chaque tranche d'âge, qui révèle la distinction progressive des relations pouvant s'établir parmi les évènements (postériorité, antériorité, simultanéité). Dans le groupe I, on trouve un usage quasiment exclusif des adverbes «poi-puis» et «dopo-après» à travers lesquels les enfants de ce groupe expriment seulement la postériorité en racontant les évènements dans un ordre séquentiel, indépendamment de la relation réelle qui les relie. Les enfants des deux autres groupes, en revanche, utilisent une plus grande variété de formes, qui permet d'exprimer les relations d'antériorité ( prima che - avant que») ainsi que de simultanéité («mentre - tandis que»). Cela confirme les résultats des études susmentionnées sur l'évolution de la capacité à structurer un récit. 
Les connecteurs métatextuels et interactifs nous amènent sur le plan plus proprement pragmatique de la production textuelle, centré sur l'aspect interactif de la communication.

S'agissant d'abord des connecteurs métatextuels, il faut préciser que dans cette catégorie on trouve deux types de signaux discursifs : les démarcatifs (qui servent à marquer l'articulation interne des différentes parties du texte, tels que «allora - alors» qui est utilisé comme formule d'ouverture ainsi que pour signaler d'autres parties du texte, «basta-c'est fini», utilisé par contre comme formule de clôture), et les reformulatifs (qui servent à signaler des phénomènes de reformulation ou d'autocorrection, tels que «voglio dire - je veux dire») [Bazzanella, 1991, 1994]. À la lueur de notre analyse, il apparait que les enfants des groupes II et III utilisent les deux types de connecteurs, tandis que les enfants du groupe I utilisent exclusivement les premiers.

En ce qui concerne les démarcatifs, on a constaté que les enfants du groupe I les utilisent seulement au début ou à la fin de leur discours, ce qui est cognitivement plus facile, tandis que les autres enfants les utilisent pour délimiter aussi d'autres parties composant l'articulation du texte, comme le début d'une séquence informative particulière (comme un flashback), la reprise du flux discursif après une interruption, le début de la seconde partie du récit. Cet usage plus différencié des démarcatifs chez les enfants les plus âgés reste cohérent avec le constat de l'évolution progressive d'une conscience métatextuelle qui ne semble pas apparaitre avant 6 ans (Gombert, 1990) et de la capacité à structurer et à planifier le texte narratif (études susmentionnées).

Pour ce qui concerne les reformulatifs, leur usage chez les enfants entre 6 et 10 ans manifeste un niveau de compétence supérieur de ces enfants à contrôler leur production et à gérer les éventuelles difficultés (comme une erreur dans un choix lexical ou dans l'ordre de succession des évènements), afin de guider l'interlocuteur dans l'interprétation du message.

Cet aspect émerge aussi dans l'usage des connecteurs interactifs au sein desquels on a identifié deux types de signaux discursifs : les éléments de remplissage (qui servent à maintenir le tour de parole, comme les expressions phrastiques «come si chiama? - comment dire?», «diciamo - disons») et les mécanismes de modulation (qui servent à renforcer ou à atténuer l'acte linguistique, comme les expressions phrastiques «diciamo - disons ${ }^{4} », ~ « m i ~ s e m b r a-$ il me semble»

4. Il faut préciser qu'un même signal discursif, comme l'expression «diciamodisons», peut avoir soit la fonction de connecteur métatextuel soit celle de 
(Bazzanella, 1991, 1994). Dans nos données, les premiers sont utilisés par les enfants des trois groupes, tandis que les mécanismes de modulation sont observés uniquement dans les tranches d'âges II et III.

Or l'usage des éléments de remplissage, bien qu'il signale une difficulté dans l'élaboration de l'énoncé, révèle aussi la capacité croissante à maintenir et gérer le rôle de locuteur et, en l'espèce, de narrateur (de fait, leur nombre augmente : 3 occurrences dans le groupe I; 8 dans le groupe II ; 13 dans le groupe III).

L'usage des mécanismes de modulation est plus intéressant encore : étant donné la fonction de ces signaux discursifs, leur utilisation révèle non seulement la capacité de contrôler sa propre production, mais aussi celle de fournir une clé de lecture à l'interlocuteur, ce qui comporte, en même temps, la capacité à considérer le point de vue de l'interlocuteur. L'accroissement de l'usage de l'ensemble des connecteurs interactifs révèle donc une meilleure compétence à fournir interprétations et évaluations et à commenter son discours, confirmant ce qui est indiqué dans la littérature.

\section{Analyse des gestes}

L'analyse relative à la composante gestuelle des récits a montré, avant tout, un accroissement progressif du nombre moyen des gestes qui passe du simple au triple du groupe I au groupe III (tableau 8) :

\begin{tabular}{|c|c|}
\hline Groupe & Nombre moyen de gestes \\
\hline I & 12,6 \\
\hline II & 27 \\
\hline III & 36,8 \\
\hline
\end{tabular}

Tableau 8. - Nombre moyen de gestes produits dans chaque groupe.

En rapportant le nombre de gestes au nombre de propositions, on constate, en outre, que la production des gestes augmente proportionnellement à celle des propositions : au fil de l'âge, le taux de gestes par proposition augmente graduellement (fig. 2) :

connecteur interactif. La distinction des deux fonctions peut se baser sur différents critères, comme l'intonation, la présence de pauses, la production simultanée d'un geste qui fonctionne comme un commentaire des paroles. 


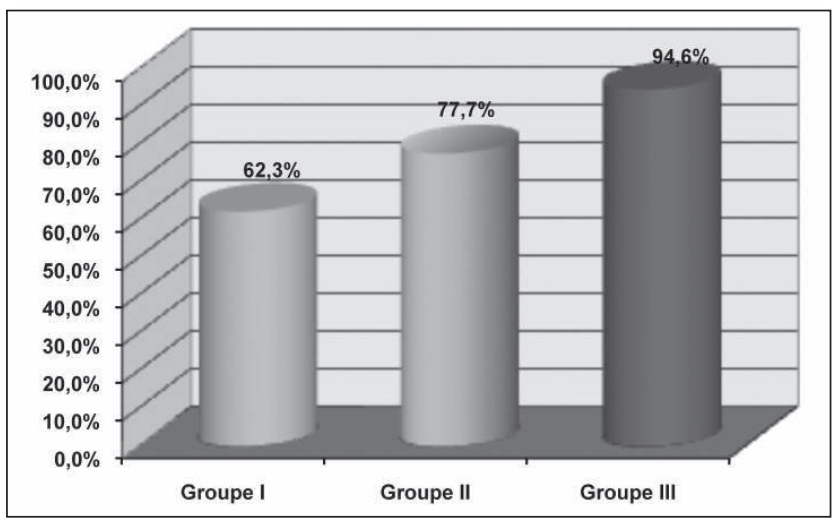

Figure 2. - Taux de gestes par proposition dans chaque groupe d'âge.

Pour ce qui concerne la distribution des gestes référentiels et pragmatiques, l'analyse a produit les résultats suivants (fig. 3) :

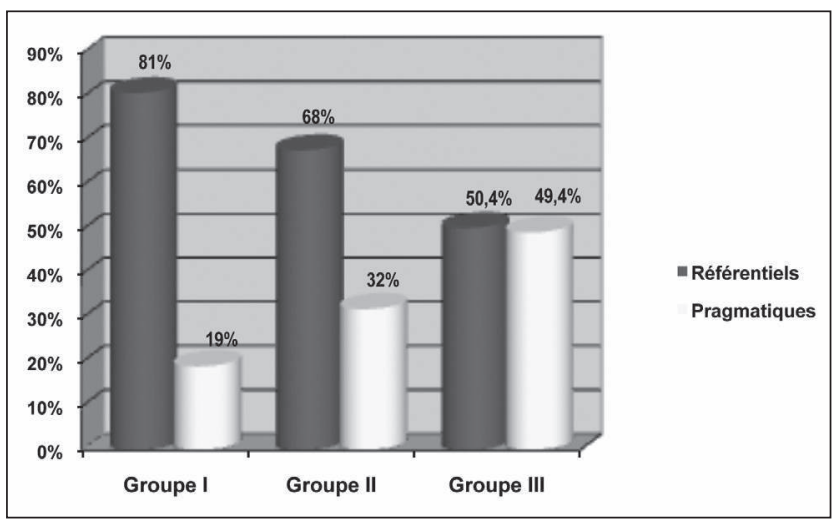

Figure 3. - Pourcentage des deux catégories de gestes par rapport au total des gestes produits dans chaque groupe.

Il est évident que la différence dans l'usage des deux catégories de gestes est très marquée parmi les trois groupes d'enfants : dans le premier, on constate une nette majorité de gestes référentiels, qui restent encore prépondérants dans le groupe II; dans le dernier groupe, par contre, on relève une distribution plus homogène entre les deux catégories de gestes, qui occupent chacune la moitié des occurrences. Ces résultats convergent avec ceux obtenus par Colletta et al. (2010) qui ont constaté une augmentation des gestes parsing et modals (que les 
auteurs appellent discursifs et de cadrage) chez les enfants de 10 ans par rapport aux enfants de 6 ans ainsi que chez les adultes.

Cette transition d'un usage clairement dominant de la gestualité référentielle à un emploi plus équilibré entre celle-ci et la gestualité pragmatique reflète l'évolution des habiletés textuelles et communicatives identifiées sur le plan linguistique. Cette corrélation apparait clairement dans l'analyse qualitative des gestes pragmatiques (performatives, modals et parsing) pour lesquels on observe des différences dans leur usage parmi les trois groupes d'âge.

\section{Analyse qualitative des gestes à fonction pragmatique}

Les différences les plus remarquables ont été relevées, en particulier, dans l'usage des modals et des parsing. Pour ce qui concerne les performatives, par contre, on n'en a pas identifié. Cependant, il semble utile d'offrir une illustration de ce type de geste pour mettre en évidence la connexion entre leur usage et la compétence communicative qu'il requiert :

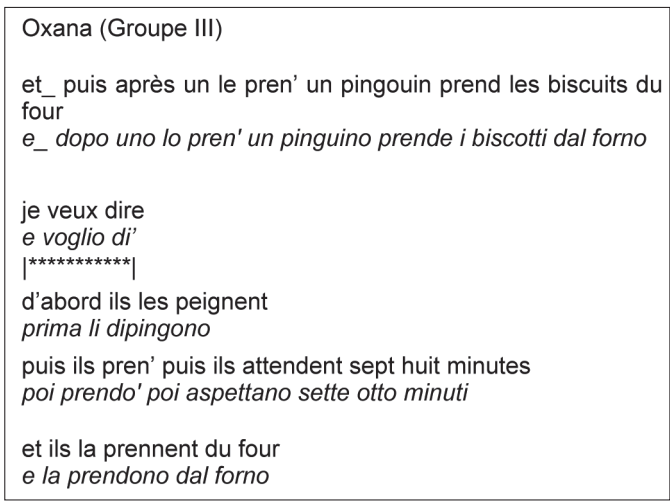

La fillette s'est trompée sur la séquence des évènements et opère une autocorrection. Celle-ci est signalée à la fois par le signal discursif qui fait fonction de reformulatif «voglio di' - je veux dire» et le geste de négation de la tête qui l'accompagne, qui sert à nier et à «effacer» (Kendon, 2002) ce qu'elle vient de dire. Dans ce cas, comme dans tous les exemples de performatives trouvés dans le corpus, ce geste n'est pas produit comme faisant partie du texte narratif mais pour exprimer un commentaire sur la production verbale, un usage qui suppose une 
compétence pragmatique qui se manifeste encore mieux dans l'emploi des modals.

S'agissant de ce type de gestes, en premier lieu, il faut rappeler que leur fonction est de fournir le cadre interprétatif de l'énoncé ou de l'unité du discours dont ils font partie (Kendon, 2004). Tout comme l'expression verbale d'évaluations et d'interprétations, donc, l'emploi des modals implique lui aussi, la capacité du locuteur à manifester son attitude mentale envers ce qu'on dit, en révélant son propre point de vue tout en considérant celui de l'interlocuteur.

On a montré que les types de verbalisations évaluatives s'accroissent, surtout à l'âge scolaire (Berman et Slobin, 1994; Baumgartner et al., 2000). De même, l'analyse de ces gestes a mis en lumière que les types de formes gestuelles utilisées avec cette fonction et les types d'informations qu'elles véhiculent se diversifient au fil de l'âge.

On a constaté que les enfants du groupe I utilisent, avec la fonction modale, une seule forme gestuelle (celle que Kendon appelle $P L^{5}$ ), dans un contexte unique et pour exprimer un seul type de signifié. Comme le montre l'exemple qui suit, ce geste accompagne la dernière proposition du récit de l'enfant (qui se réfère à l'image finale du dessin animé où l'on voit l'arbre de Noël décoré) et il sert à communiquer que le locuteur veut se retirer du «champ d'action» (Kendon, 2004) et invite donc l'interlocuteur à considérer son récit comme terminé :

(4)

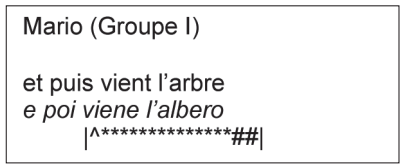

À partir de la tranche d'âge intermédiaire, l'usage des gestes modals devient plus diversifié, tant dans leur forme que dans leur signifié, comme l'illustrent les deux exemples suivants :

5. Il s'agit d'un geste qui appartient à un groupe de gestes que l'auteur appelle famille Open Hand Supine qui se caractérise par la main ouverte et la paume orientée vers le haut, et qui comporte trois types de mouvements sur la base desquels l'auteur a distingué trois types d'expressions gestuelles. Le $P L$ ou geste avec un mouvement latéral est caractérisé précisément par un mouvement latéral accompli avec le bras. Kendon (2004) relève cette expression gestuelle dans plusieurs contextes discursifs. 
(5)

Chiara (Groupe II)
et alors (...) puis ils ont mis puis ils ont fait l'arbre de ne (.) un
arbre
e allora (...) poi hanno mes hanno fatto un albero di ne (.) un
albero
où ils ont mis beaucoup de petites boules (.) le papa et la mère (..)
che hanno messo ta_nte tante palline (.) il babbo e la madre (..)
puis (...) puis ils ont ouvert tous les cadeaux
poi (...) poi hanno aperto tutti i regali
\[ \left.\right|^{* * * * * \mid} \]

Dans le rappel des évènements racontés, la fillette semble avoir des moments d'hésitation qui se manifestent par les pauses produites dans les propositions tout comme par le geste, exécuté en soulevant les épaules, qui signale à l'interlocuteur qu'elle n'est pas sure de la séquence des évènements. Il sert, donc, à fournir la clé interprétative de l'énoncé.

(6)

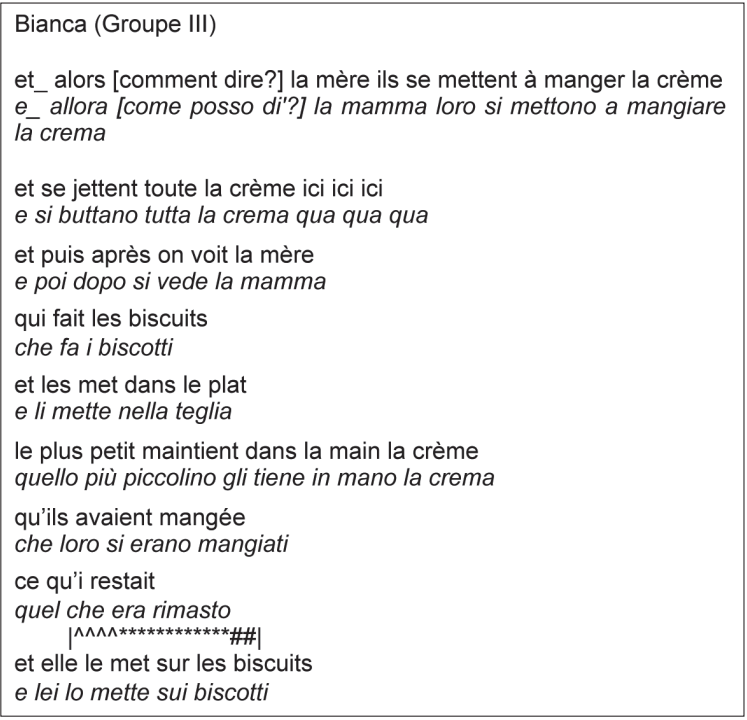

Dans cet extrait $\mathrm{B}$ utilise un geste $P L$ au moment où elle ajoute un commentaire en se référant à la crème déjà mentionnée auparavant. Ce geste sert à communiquer que le contenu de son énoncé est évident (un emploi largement attesté par Kendon) : ayant dit que les pingouins avaient mangé la crème, il est clair que la crème que maman-pingouin 
peut mettre sur les biscuits est «quel che era rimasto - ce qui restait». Cette idée d'évidence n'est pas exprimée dans le message verbal mais apparait à travers le geste qui, tout comme l'intonation, fournit la clé interprétative de l'énoncé, en contribuant de cette façon à exprimer son signifié global.

Comme on le voit, ces derniers usages requièrent la capacité à commenter et contrôler sa propre production, ainsi que la capacité à gérer la relation avec l'interlocuteur pour maintenir son attention. On peut donc en conclure que l'habileté à utiliser ces gestes est conforme à l'évolution de la capacité à exprimer des commentaires, des évaluations et à interpréter les évènements racontés déjà constatée dans la dimension linguistique.

En ce qui concerne, enfin, les gestes de parsing, il faut rappeler que leur fonction est de marquer les différentes parties du discours (Kendon, 2004). On a pu constater que les enfants les plus jeunes utilisent ce type de gestes exclusivement pour marquer les différentes unités qui constituent une liste. Il s'agit des gestes conventionnels de numération qui scandent la désignation de chaque élément de la liste (comme celles des cadeaux dans l'exemple) et qui sont réalisés à travers l'ouverture successive des doigts :

(7)

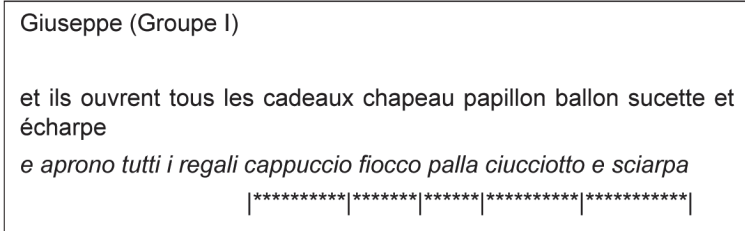

La limitation à ce type de contexte discursif (énumération), qui se présente comme un type de structure textuelle très simple à réaliser, reste conforme à la faible capacité des enfants de cet âge à concevoir le discours comme une unité globale. De fait, comme on l'a vu dans l'analyse des connecteurs, leurs narrations semblent dépourvues de structuration interne.

Les enfants les plus âgés, par contre, utilisent les gestes de parsing pour signaler l'articulation interne de leur récit, comme l'ouverture du discours, l'introduction des personnages, le passage à une nouvelle séquence narrative ou la reprise du flux narratif après l'intrusion d'un flashback. Ces gestes, en outre, se trouvent souvent en co-occurrence 
avec les connecteurs qui servent justement à marquer la structure du texte et contribuent de cette façon à la segmentation du discours.

Le premier exemple illustre l'usage d'un geste de parsing pour marquer le début du récit. Il s'agit de la version d'une forme gestuelle identifiée par Kendon ${ }^{6}$ et à laquelle il attribue (inspiré par Calbris, 2003) le «thème sémantique» de «établir les confins/limites de quelque chose». Le geste accompagne le démarcatif «allora - alors» utilisé comme une formule d'ouverture (Bazzanella, 1991, 1994) :

(8)

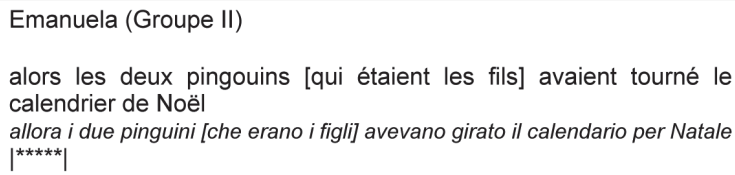

Dans l'exemple qui suit, l'usage du même geste, produit avec le même démarcatif, sert à marquer, tout comme le connecteur, le début d'une séquence narrative différente par rapport à celle qui précède (ici la seconde partie) :

(9)

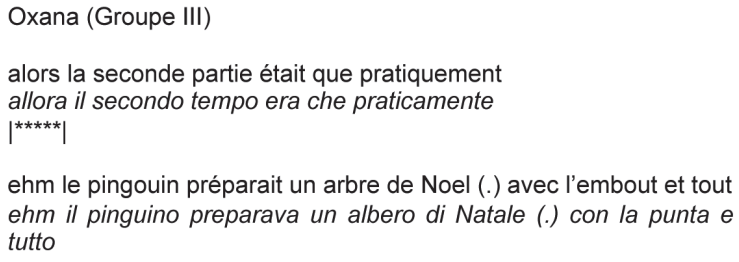

L'exemple suivant montre un geste de parsing produit avec la main ouverte et la paume vers le haut (ce que Kendon appelle $P P^{7}$ ) pour signaler la première mention des personnages :

6. Il s'agit d'un geste qui dans la description de l'auteur ainsi que de Calbris (2003) est produit avec les mains ouvertes, les paumes l'une face à l'autre, qui se soulèvent et s'abaissent dans un mouvement au tracé net. Dans la version qu'en donne à voir cette fillette, le geste n'est pas si précis.

7. Il s'agit d'une autre expression gestuelle appartenant à la famille Open Hand Supine que Kendon (2004) appelle gestes avec les paumes en présentation $(P P)$, généralement accomplis avec une seule main qui s'abaisse une fois. 
(10)

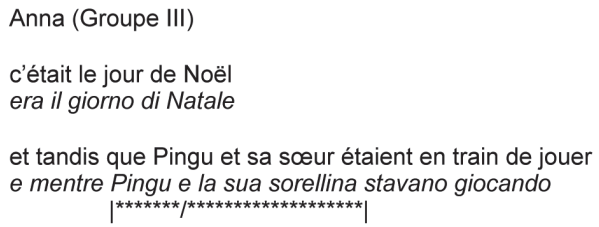

Dans le dernier exemple, le geste de parsing sert à signaler la reprise du flux discursif qui avait été interrompu par l'intrusion d'un flashback:

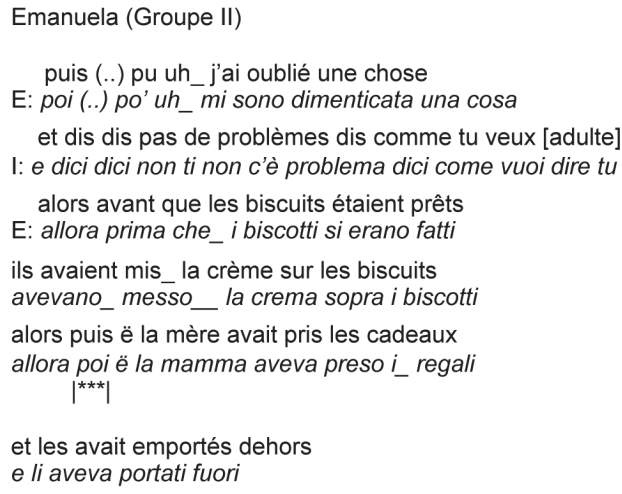

Dans ce cas, le geste, réalisé avec un mouvement de la tête qui est abaissée puis repoussée vers l'arrière, n'est pas produit avec le connecteur «allora - alors » qui signale la progression du discours, mais avec le temporel «poi - puis », qui sert à rétablir l'ordre dans lequel les évènements se développent après le flashback.

Il faut noter que ces gestes surviennent en accompagnement de propositions d'orientation (Labov et Waletzky, 1967; Labov, 1972) ou métanarratives (McNeill, 1992), c'est-à-dire de propositions dans lesquelles on précise la situation, les personnages, le lieu et le moment de l'histoire, ou dans lesquelles on se réfère explicitement à la structure du récit. L'usage de ces gestes chez les enfants les plus âgés est donc cohérent non seulement avec leur meilleure capacité à marquer linguistiquement l'organisation interne de la narration, mais aussi avec celle qui consiste à fournir les informations nécessaires pour créer un cadre narratif et qui semble se manifester justement à l'âge scolaire (Peterson et McCabe, 1983 ; Peterson, 1990 ; Berman et Slobin, 1994 ; Berman, 
2001). L'usage de ces gestes reflète donc la compétence métanarrative de ces enfants.

\section{Conclusion}

L'analyse a mis en évidence plusieurs phénomènes intéressants qui, d'une part, confirment les résultats avancés par d'autres recherches (McNeill, 1992; Colletta, 2004, 2009), d'autre part, permettent d'approfondir des aspects encore peu explorés à l'intérieur de la tranche d'âge considérée.

La comparaison des données relatives aux deux dimensions langagières montre clairement qu'il y a d'importants phénomènes parallèles dans le développement de ces deux modalités d'expression, liés à l'évolution des habiletés textuelles et pragmatico-communicatives.

Comme on l'a montré, la capacité à utiliser un plus large répertoire de connecteurs temporels à travers lesquels exprimer les différents types de relation temporelle qui lient les évènements, la capacité à utiliser les connecteurs démarcatifs pour articuler un texte en plusieurs parties et la capacité à utiliser les gestes de parsing avec cette même fonction se manifestent dans la même période. De plus, l'habileté à utiliser les gestes modals pour exprimer une plus grande variété de signifiés trouve une correspondance dans la capacité à utiliser les connecteurs qui font fonction de mécanismes de modulation et qui, comme on l'a indiqué, ne sont pas présents avant 6 ans.

La capacité à intégrer les outils linguistiques et les signes gestuels ayant la même fonction est une manifestation importante de l'évolution parallèle de ces deux modalités d'expression. Cela, rapporté à l'élaboration d'un discours narratif, témoigne de l'habileté croissante à concevoir le récit comme une unité globale, ce qui permet de réaliser la fonction référentielle de la narration, ainsi que celle à reconnaitre la nature interactive de la narration, ce qui permet d'accomplir la fonction évaluative.

La recherche effectuée semble offrir une nouvelle contribution à la compréhension de la relation établie entre gestes et paroles au cours du développement du langage. Les résultats viennent confirmer les thèses défendues par Kendon (1980, 2004) et McNeill (1992, 2000), d'après lesquelles le code linguistique et le code gestuel sont à considérer comme deux formes, différentes mais fortement intégrées, de la faculté du langage. 


\section{RÉFÉRENCES BIBLIOGRAPHIQUES}

Bamberg M. et Damrad-Frye R. (1991) : «On the ability to provide evaluative comments: Further explorations of children's narrative competencies », Journal of Child Language, vol. 18, n 3, p. 689-710.

Bamberg M. et ReIlly J. S. (1996) : «Emotion, Narrative, and Affect: How children discover the relationship between "what to say" and "how to say it" », dans D. I. Slobin, J. Gerhardt, A. Kyratzis et J. Guo (éds), Social Interaction, Social Context, and Language. Essays in Honor of Susan Ervin-Tripp, Hillsdale (NJ), Lawrence Erlbaum, p. 329-341.

Bates E., Benigni L., Bretherton I., Camaioni L. et Volterra V. (1979) : The emergence of symbols: cognition and communication in infancy, New York, Academic Press.

Bates E., Camaioni L. et Volterra V. (1975) : «The acquisition of performatives prior to speech », Merrill-Palmer Quarterly, vol. 21, $\mathrm{n}^{\circ} 3$, p. 203-226.

Baumgartner E., Devescovi A. et D'Amico S. (2000) : Il lessico psicologico. Origine ed evoluzione, Rome, Carocci.

Bazzanella C. (1991) : «I segnali discorsivi», dans L. Renzi, G. Salvi et A. Cardinaletti (éds), Grande grammatica italiana di consultazione, Bologne, Il Mulino, p. 225-257.

BazzANella C. (1994) : Le facce del parlare: un approccio pragmatico all'italiano parlato, Florence, La Nuova Italia.

Berman R. A. (2001) : «Setting the Narrative Scene: How Children Begin to Tell a story», dans K. E. Nelson, A. Aksu-Koç et C. E. Johnson (éds), Children's Language. Vol. 10: Developing Narrative and Discourse Competence, Mahwah (New Jersey), Lawrence Erlbaum.

Berman R. A. et Slobin D. I. (1994) : Relating events in narrative: A crosslinguistic developmental study, Hillsdale (NJ), Lawrence Erlbaum Associates.

BerRetTA M. (1984) : «Connettivi testuali in italiano e pianificazione del discorso», dans L. Coveri (éd.), Linguistica testuale. Atti del XV Congresso internazionale di studi della Società di linguistica italiana (Gênes - Santa Margherita Ligure, 8-10 mai 1981), Rome, Bulloni, p. 237-254.

Butcher C. et Goldin-Meadow S. (2000) : «Gesture and the transition from one-to two-word speech: when hand and mouth come together", dans D. McNeill (éd.), Language and gesture, Cambridge, Cambridge University Press, p. 235-257.

CAlbRis G. (2003) : «From cutting an object to a clear cut analysis: Gesture as the representation of a preconceptual schema linking concrete actions to abstract notions», Gesture, vol. 3, n 1, p. 19-46. 
Capirci O., Contaldo A., Caselli M. C. et Volterra V. (2005) : «From action to language through gesture: A longitudinal perspective», dans K. Liebal, C. Müller et S. Pika (éds), Gestural Communication in Nonhuman and Human Primates, numéro special de Gesture, vol. 5, n 1/2, p. 155-177.

Capirci O., Cristilli C., De Angelis V. et Graziano M. (à paraitre) : «Learning to use gesture in narratives: developmental trends in formal and semantic gesture competence», dans G. Stam et M. Ishino (éds), Integrating Gestures, John Benjamins.

Capirci O., Iverson J. M., Pizzuto E. et Volterra V. (1996) : «Gestures and words during the transition to two-word speech», Journal of Child Language, vol. 23, $\mathrm{n}^{\circ} 3$, p. 645-673.

Chini M., Ferraris S., V alentini A. et Businaro B. (2003) : «Aspetti della testualità», dans A. Giacalone Ramat (éd.), Verso l'italiano. Percorsi e strategie di acquisizione, Rome, Carocci, p. 178-219.

Colletta J.-M. (2004) : Le développement de la parole chez l'enfant âgé de 6 à 11 ans. Corps, langage et cognition, Hayen, Mardaga.

Colletta J.-M. (2009) : «Comparative analysis of children's narratives at different ages: A multimodal approach», Gesture, vol. 9, nº 1, p. 61-97.

Colletta J.-M., PellenQ C. et Guidetti M. (2010) : «Age-related changes in co-speech gesture and narrative: Evidence from French children and adults », Speech Communication, vol. 52, nº 6, p. 565-576.

Cristilli C., Capirci O. et Graziano M. (à paraitre) : «Le funzioni anaforiche della gestualità nel racconto dei bambini», dans M. Pettorino, F. Albano Leoni, I. Chiari, F. Dovetto et A. Giannini (éds), La Comunicazione Parlata. Atti del III Congresso Internazionale GSCP (Naples, 23-25 février 2009).

Gombert J. E. (1990) : Le développement métalinguistique, Paris, Presses universitaires de France.

Halliday M. A. K. et Hasan R. (1976) : Cohesion in English, Londres, Longman.

Iverson J. M. et FAGAN M. K. (2004) : «Infant vocal-motor coordination: Precursor to the gesture-speech system?», Child Development, vol. 75, $n^{\circ} 4$, p. 1053-1066.

Iverson J. M. et Thelen E. (1999) : «Hand, Mouth and Brain: the Dynamic Emergence of Speech and Gesture», Journal of Consciousness Studies, vol. $6, \mathrm{n}^{\circ} 11-12$, p. 19-40.

Iverson J. M., Capirci O. et Caselli M. C. (1994) : «From communication to language in two modalities », Cognitive Development, vol. $9, \mathrm{n}^{\circ} 1$, p. 23-43. 
Kendon A. (1972) : «Some relationships between body motion and speech», dans A. Seigman et B. Pope (éds), Studies in Dyadic Communication, Elmsford, New York, Pergamon Press, p. 177-216.

Kendon A. (1980) : «Gesture and speech: two aspects of the process of utterance», dans M. R. Key (éd.), Nonverbal Communication and Language, La Haye, Mouton, p. 207-227.

Kendon A. (2002) : «Some uses of head shake», Gesture, vol. 2, n 2 , p. $147-182$.

Kendon A. (2004): Gesture: Visible Action as Utterance, Cambridge, Cambridge University Press.

LaBov W. (1972) : Language in the inner city: Studies in the Black English Vernacular, Oxford, Basil Blackwell.

LABOv W. et WaLETZKY J. (1967) : «Narrative analysis: Oral versions of personal expérience», dans J. Helm, Essays on the verbal and visual arts, Seattle, University of Washington Press, p. 12-44.

LiszKowsKi U. (2005) : «Human twelve-month-olds point cooperatively to share interest with and helpfully provide information for a communicative Partner», Gesture, vol. 1, nº 2, p. 135-154.

Losh M., Bellugi U., Reilly J. S. et Anderson D. (2000) : «Narrative as a social engagement tool: the excessive use of evaluation in narratives from children with Williams syndrome», Narrative inquiry, vol. 10, $\mathrm{n}^{\circ} 2$, p. 265-299.

LundQuist L. (1994) : La Cohérence textuelle: syntaxe, sémantique, pragmatique, Frederiksberg, Samfundslitteratur.

Mandler J. M. et Johnson N. S. (1977) : «Remembrance of things parsed. Story structure and recall», Cognitive psychology, n 9, p. 111-191.

MCNeILL D. (1992): Hand and Mind: What gestures reveal about thought, Chicago, Chicago University Press.

McNeill D. (éd.) (2000) : Language and gesture, Cambridge, Cambridge University Press.

ÖZçalişKan S. et Goldin-Meadow S. (2005) : «Gesture is at the cutting edge of early language development», Cognition, vol. 96, $\mathrm{n}^{\circ} 3$, p. B101-B113.

Peterson C. (1990) : «The who, when and where of early narratives», Journal of Child Language, vol. 17, n 2 2, p. 433-455.

Peterson C. et McCabe A. (1983): Developmental psycholinguistics: Three ways of looking at a child's narrative, New York, Plenum Press.

Peterson C. et McCabe A. (1987) : «The connective "and": do older children use it less as they learn other connectives?», Journal of Child Language, vol. 14, $\mathrm{n}^{\circ} 2$, p. 375-381. 
Pizzuto E., Capobianco M. et Devescovi A. (2005) : «Gestural-vocal deixis and representational skills in early in early language development», Interaction Studies, vol. 6, n 2, p. 223-252.

Reig Alamillo A. et Colletta J.-M. (soumis) : «Apprendre à raconter, apprendre à commenter».

ReILly J. S. (1992) : «How to tell a good story: The intersection of language and affect in children's narratives », Journal of Narrative and Life History, vol. 2, no 4, p. 355-377.

Reilly J. S., Losh M., Bellugi U. et Wulfeck B. (2004) : “"Frog, here are you?" Narratives in children with specific language impairement, early focal injury and Williams syndrome», Brain and language, vol. 88, no 2 , p. 229-247.

Roulet E., Auchlin A., Moeschler J., Rubattel C. et Scheling M. (1985) : L'articulation du discours en français contemporain, Berne, Peter Lang. Simone R. (1990) : Fondamenti di linguistica, Bari, Laterza.

Stein N. L. et Glenn C. G. (1979) : «An Analysis of Story Comprehension in Elementary School Children », dans R. O. Freedle (éd.), New Directions in Discourse Processing, Norwood, Ablex, p. 53-119.

Tomasello M. (2003) : Constructing a Language: A Usage-Based Theory of Language Acquisition, Cambridge (Massachusetts), Harvard University Press.

\section{APPENDICE}

La transcription verbale reflète le plus fidèlement possible la réelle prononciation du locuteur. On a opté pour la graphie orthographique habituelle, sans l'usage de signes de ponctuation, à l'exception du point interrogatif et du point d'exclamation. Des conventions ont été adoptées pour :

- les sons d'hésitation (représentés par «hem», «mh»);

- les allongements vocaliques (signalés par_ $\_$);

- les pauses (représentées par (.), (..) et (...), qui correspondent, respectivement, aux pauses brèves, longues et très longues);

- la voyelle indistincte moyenne (shwa) (représentée par «ё»).

Pour la transcription des gestes, on a adopté le système élaboré par Kendon $(1980,2004)$ pour représenter les phases identifiées dans l'exécution des gestes : préparation (phase préparatoire pendant laquelle la main se déplace pour accomplir le geste); stroke (partie «significative» du mouvement); recovery (phase de retour à la position initiale ou à un nouvel emplacement); post-stroke hold (suspension du mouvement dans la position rejointe dans le stroke). Les symboles utilisés sont les suivants : 
_ $\wedge \wedge \wedge$ indique la phase de préparation;

_ **** indique le stroke;

_ ***** indique le post-stroke hold;

- \#\#\# indique la phase de retour;

- I délimite la séquence gestuelle.

La transcription du geste est placée sur la ligne en dessous de la transcription verbale, alignée le plus possible avec la séquence verbale à laquelle le geste est associé. 